University of Nebraska - Lincoln

DigitalCommons@University of Nebraska - Lincoln

$12-21-2010$

Detecting Electron Motion in Atoms and Molecules

\author{
Hua-Chieh Shao \\ University of Nebraska-Lincoln, hshao@unl.edu \\ Anthony F. Starace \\ University of Nebraska-Lincoln, astarace1@unl.edu
}

Follow this and additional works at: https://digitalcommons.unl.edu/physicsstarace

Part of the Physics Commons

Shao, Hua-Chieh and Starace, Anthony F., "Detecting Electron Motion in Atoms and Molecules" (2010). Anthony F. Starace Publications. 178.

https://digitalcommons.unl.edu/physicsstarace/178

This Article is brought to you for free and open access by the Research Papers in Physics and Astronomy at DigitalCommons@University of Nebraska - Lincoln. It has been accepted for inclusion in Anthony F. Starace Publications by an authorized administrator of DigitalCommons@University of Nebraska - Lincoln. 


\title{
Detecting Electron Motion in Atoms and Molecules
}

\author{
Hua-Chieh Shao and Anthony F. Starace \\ Department of Physics and Astronomy, The University of Nebraska, Lincoln, Nebraska 68588-0299, USA
}

(Received 24 July 2010; published 20 December 2010)

\begin{abstract}
The detection of spatial and temporal electronic motion by scattering of subfemtosecond pulses of $10 \mathrm{keV}$ electrons from coherent superpositions of electronic states of both $\mathrm{H}$ and $T_{2}^{+}$is investigated. For the $\mathrm{H}$ atom, we predict changes in the diffraction images that reflect the time-dependent effective radius of the electronic charge density. For an aligned $T_{2}^{+}$molecule, the diffraction image changes reflect the timedependent localization or delocalization of the electronic charge density.
\end{abstract}

DOI: 10.1103/PhysRevLett.105.263201

PACS numbers: $34.80 . \mathrm{Bm}$

At present, attosecond physics is able to provide unprecedented temporal resolution and control of electronic processes [1,2]. Single, few-cycle attosecond pulses with photon energies $\hbar \omega<100 \mathrm{eV}$ and temporal widths in the range of 130-80 as [3,4] have been achieved, making possible the new field of light-wave electronics in which the electric field of the pulse (rather than its intensity profile) is used to control electronic motion [2,5,6]. However, to obtain spatial resolution of temporal changes in electronic charge densities, pulses having much shorter wavelengths are necessary. Ultrafast electron diffraction [7-9] and ultrafast electron microscopy [8,10-13] have also made significant progress recently, enabling the observation of transient structures in chemical reactions and of time-dependent phenomena in condensed matter. The temporal widths of these electron pulses, however, range from femtosecond to picosecond, which is inadequate for studying electronic motion in atoms and molecules. Recently, methods have been proposed for producing single-electron, attosecond pulses [14-16]. The attosecond temporal and subangstrom spatial resolutions of such short $\mathrm{keV}$ electron pulses (at the target location) would make the four-dimensional (4D) study of electronic motion feasible.

We report here benchmark calculations for attosecond electron pulse scattering from oscillating electronic charge distributions in two prototypical systems: the $\mathrm{H}$ atom and the $T_{2}^{+}$molecule. We demonstrate the effect of such spatial and temporal charge oscillations on the differential scattering cross section (DSCS). For the $\mathrm{H}$ atom, a fewfemtosecond laser pulse is proposed to excite a coherent superposition of two electronic states; for the $T_{2}^{+}$molecule, such a superposition state is assumed. In both cases the charge distribution oscillates with the beat frequency. The DSCSs for an electron energy of $10 \mathrm{keV}$ are calculated in the Born approximation. For the $\mathrm{H}$ atom, the charge distribution has an oscillating effective radius. For the $T_{2}^{+}$ molecule, the superposed states have an electronic charge density that oscillates from one nucleus to the other. In both cases, the DSCSs are predicted to exhibit the 4D motion of the electronic charge density.
Figure 1 shows the setup [8,9] for which our $\mathrm{H}$ atom calculations are performed. The few-femtosecond laser pulse creates a superposition of electronic states which is then probed by a time-delayed, $10 \mathrm{keV}$ single-electron attosecond pulse. The electron pulse may be generated by photoemission $[15,17]$ and then compressed to an attosecond duration by a spatially dependent ponderomotive potential $[15,16]$. By adjusting the time delay between the pump and the probe pulses, the 4D motion of the oscillating charge distribution is manifested in the time-resolved diffraction images of the scattered electrons.

For the $\mathrm{H}$ atom, the energy of the 8th harmonic of the Ti: sapphire laser relative to the $1 s$ ground state is between the energies of the $3 p$ and $4 p$ states. The populations of these and nearby states can be calculated by using first-order time-dependent perturbation theory for a pulse whose vector potential has the form

$$
\mathbf{A}(t)=\boldsymbol{\epsilon} A_{0} \exp \left[-4(\ln 2) t^{2} / \tau^{2}\right] \sin (\omega t+\phi),
$$

where $\boldsymbol{\epsilon}$ is the polarization vector, $A_{0}$ is the amplitude, $\tau$ is the width at half height of the Gaussian envelope, $\omega$ is the carrier frequency, and $\phi$ is the carrier-envelope phase. In the electric dipole approximation and by defining $\bar{\tau}^{2} \equiv$ $\tau^{2} / 8 \ln 2$, the transition probability for $|i\rangle \rightarrow|f\rangle$ is

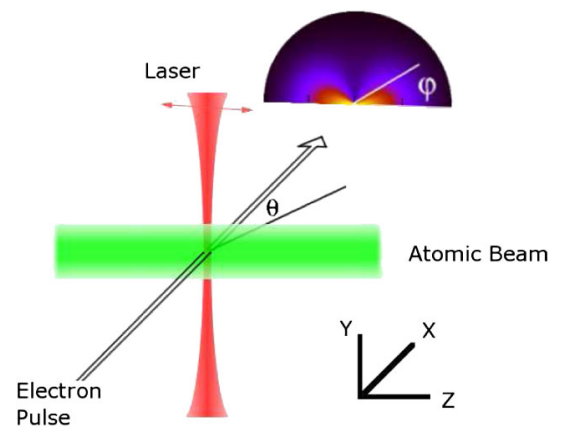

FIG. 1 (color online). Proposed $\mathrm{H}$ atom experiment. The red arrow indicates the polarization of the pump laser. For future reference, the coordinates and the angles are defined in the figure. 


$$
\begin{aligned}
|\langle f|\tilde{T}| i\rangle|^{2}= & \frac{\pi}{2} \alpha^{2}\left(A_{0} \omega_{f i}\right)^{2}|\langle f|\boldsymbol{\epsilon} \cdot \mathbf{r}| i\rangle|^{2} \bar{\tau}^{2}\left[e^{-\left(\omega-\omega_{f i}\right)^{2} \bar{\tau}^{2}}\right. \\
& \left.+e^{-\left(\omega+\omega_{f i}\right)^{2} \bar{\tau}^{2}}-2 \cos 2 \phi e^{-\left(\omega^{2}+\omega_{f i}^{2}\right) \bar{\tau}^{2}}\right],
\end{aligned}
$$

where $\alpha$ is the fine-structure constant and $\omega_{f i} \equiv E_{f}-E_{i}$. (Atomic units are used throughout this Letter.)

The populations of several $n p$ states produced by a linearly polarized laser pulse with central energy $12.45 \mathrm{eV}$ and peak intensity $I=1 \times 10^{12} \mathrm{~W} / \mathrm{cm}^{2}$ as a function of the pulse width $\tau$ are shown in Fig. 2. (Since there are many cycles per pulse, we have set $\phi=0$.) For $\tau=8.7 \mathrm{fs}$, the $3 p$ and $4 p$ states are equally populated, while the populations of other states are negligible. The charge density of the resulting coherent state $\mathrm{H}^{*}(3 p+4 p)$ is

$$
\rho(t)=\frac{1}{2}\left[\phi_{3 p_{0}}^{2}+\phi_{4 p_{0}}^{2}+2 \phi_{3 p_{0}} \phi_{4 p_{0}} \cos \left(\omega_{43} t\right)\right] .
$$

The interference term oscillates with the beat frequency $\omega_{43}$ (with a period of $6.3 \mathrm{fs}$ ), resulting in an oscillating effective radius of the charge distribution.

For a keV electron pulse, the DSCSs for the $\mathrm{H}$ atom may be calculated in the first-order Born approximation [18]. Since the temporal width of the ultrafast electron pulse $(\approx$ 110 as) is orders of magnitude shorter than the beat period (6.3 fs), the atomic charge density is treated as frozen in time during the scattering of the electron pulse. Moreover, electron exchange is neglected owing to the high energy of the electron pulse and its small momentum transfer [19]. The elastic scattering amplitude is

$$
f(\mathbf{q} ; t)=-\frac{m}{2 \pi} \int d \mathbf{r}^{\prime} e^{-i \mathbf{q} \cdot \mathbf{r}^{\prime}} V\left(\mathbf{r}^{\prime} ; t\right),
$$

where $\mathbf{q}$ is the momentum transfer and $m$ is the reduced mass. The effective potential $V\left(\mathbf{r}^{\prime} ; t\right)$ between the incident electron and the $\mathrm{H}$ atom at the origin is

$$
V(\mathbf{r} ; t)=-\frac{1}{|\mathbf{r}|}+\int d \mathbf{r}^{\prime} \frac{\rho\left(\mathbf{r}^{\prime} ; t\right)}{\left|\mathbf{r}-\mathbf{r}^{\prime}\right|}
$$

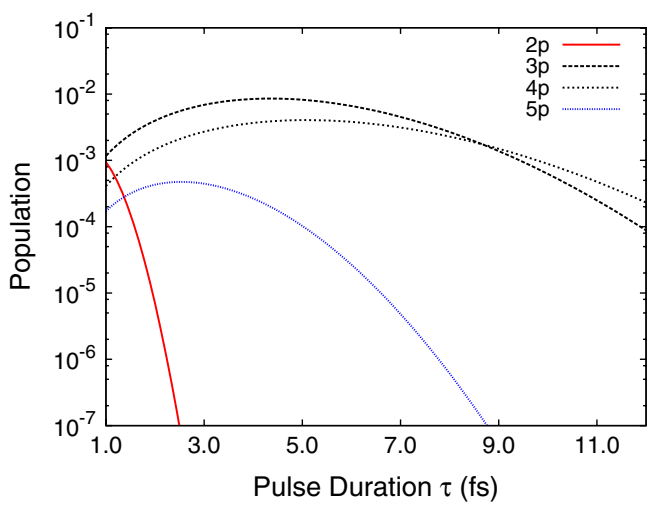

FIG. 2 (color online). Populations of $n p$ states of the $\mathrm{H}$ atom as a function of the duration of a laser pulse with peak intensity $I=1 \times 10^{12} \mathrm{~W} / \mathrm{cm}^{2}, \omega=12.45 \mathrm{eV}$, and $\phi=0$ [cf. Eq. (1)]. where the electron density $\rho$ is defined in Eq. (3). The DSCS is averaged over the momentum distribution $|a(\mathbf{k})|^{2}$ of the electron pulse, i.e.,

$$
\frac{d \sigma}{d \Omega}=\int d \mathbf{k}|a(\mathbf{k})|^{2}|f(\mathbf{q} ; t)|^{2} .
$$

For a well-collimated pulse moving in the $x$ direction with central momentum $\mathbf{p}_{0}$, the momentum distribution is

$$
|a(\mathbf{k})|^{2}=\prod_{i=x, y, z} \frac{1}{\sqrt{2 \pi} \sigma_{i}} \exp \left(-\frac{\left(k_{i}-p_{0 i}\right)^{2}}{2 \sigma_{i}^{2}}\right)
$$

where $\sigma_{i}^{2}$ is its variance along each axis. We set $\sigma_{x} / p_{0 x}=$ $1.5 \times 10^{-4}$, so that $\Delta x / p_{0 x} \equiv \Delta t=\left(2 \sigma_{x} p_{0 x}\right)^{-1}=$ 110 as. The cylindrically symmetric transverse momentum distribution corresponds to an angular divergence $\pm 10^{-3} \mathrm{rad}$. The $10 \mathrm{keV}$ electron pulse (a case considered in Ref. [15]) has a de Broglie wavelength of $0.12 \AA$.

The elastic DSCSs in the detection plane (cf. Fig. 1) at three delay times are shown on the right in Fig. 3; the electron densities [cf. Eq. (3)] at those times are shown on the left. As the effective radius of the charge density increases, the DSCS increases accordingly, demonstrating the ability of an ultrafast electron pulse to map both spatial and temporal electronic motion in atoms. In the left column of Fig. 4, the DSCSs at three delay times are plotted as a function of the azimuthal angle $\varphi$ for two different polar angles $\theta$ (cf. Fig. 1). The variation of the elastic DSCSs with pump-probe delay time is in each case over $100 \%$. Note that inelastic DSCSs are largest for small scattering angles. At large polar angles, deexcitation to the $3 s$ state gives the largest contribution to the inelastic DSCS. In the
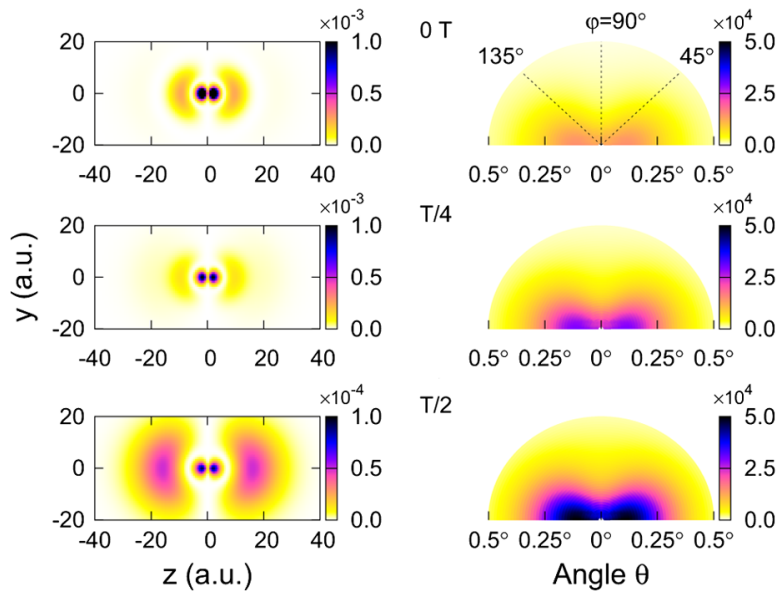

FIG. 3 (color online). Right column: Differential cross sections in the $y-z$ plane (cf. Fig. 1) for scattering of a 110 as, $10 \mathrm{keV}$ electron pulse from the coherent state $\mathrm{H}^{*}(3 p+4 p)$ (whose beat period is $T=6.3 \mathrm{fs}$ ). Left column: Charge density of the state $\mathrm{H}^{*}(3 p+4 p)$. The three rows correspond to pumpprobe time delays of $0 \mathrm{~T}, \mathrm{~T} / 4$, and $\mathrm{T} / 2$. Owing to symmetry, we show only the upper half of each diffraction image. 

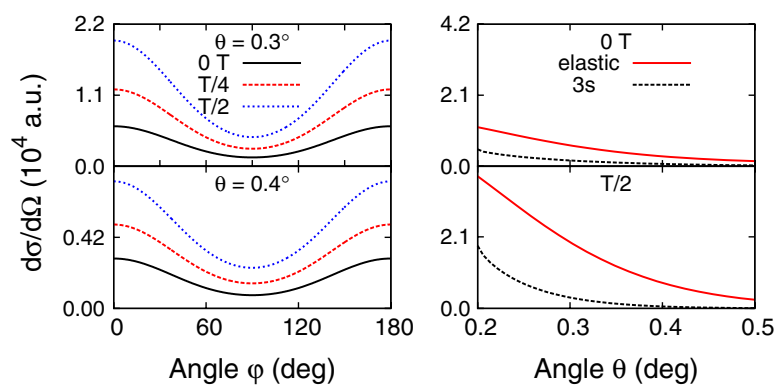

FIG. 4 (color online). Left column: Elastic DSCSs as a function of the azimuthal angle $\varphi$ for two different polar angles $\theta$ (cf. Fig. 1); each panel shows results for three pump-probe time delays. Right column: Comparison of the elastic DSCS and the most important inelastic one (for deexcitation to the $3 s$ state) plotted vs the polar scattering angle $\theta$.

right column of Fig. 4, we compare the elastic DSCS to the DSCS for this most important inelastic process as a function of polar angle $\theta$ for $\varphi=0^{\circ}$. For polar angles $\theta>$ $0.3^{\circ}$, the contribution of inelastic scattering is insignificant compared to the elastic DSCS for any pump-probe delay time.

Attosecond extreme ultraviolet pulse photoionization of coherent superpositions of electronic states of the $\mathrm{H}_{2}^{+}$ molecule have been studied theoretically [20-22]. The photoelectron probabilities exhibit asymmetries dependent on the pump-probe time delay. Only one of these studies [22] showed how to produce the coherent states and, by using a one-dimensional (1D) approximation, how nuclear motion affects the results: The electronic superposition state coherence survived even for times comparable to the beat period. Very recently, ultrashort electron pulse diffraction from the $\mathrm{H}_{2}^{+}$molecule has been investigated by using a $1 \mathrm{D}$ approximation [23]. Inelastic and exchange processes were found to play minor roles.

To illustrate attosecond electron pulse diffraction from a coherently oscillating molecular electronic state in a theoretically tractable system (that allows one to obtain an analytic expression for the DSCS), we consider the superposition of the $\sigma_{g} 1 s$ and $\sigma_{u} 1 s$ states of a transversely aligned tritium molecular ion $T_{2}^{+}$for a bond length $R=$ 6 a.u. (where the potential curves are relatively flat) [22]. This superposition state gives alternately a localized or delocalized electronic charge density that varies with a beat period of $\approx 7.9 \mathrm{fs}$ [24] and that shows the "hopping" of the electron from one nucleus to the other. The molecular states are constructed by employing the LCAO approximation using the $1 s$ state of the $T$ atom [20,21]. This approximation is valid for large bond lengths owing to little overlap of the atomic wave functions on different nuclei. The molecular states are thus given by

$$
\begin{aligned}
& \psi_{\sigma_{g} 1 s}=A_{g}\left(\phi_{1 s}^{(1)}+\phi_{1 s}^{(2)}\right), \\
& \psi_{\sigma_{u} 1 s}=A_{u}\left(\phi_{1 s}^{(1)}-\phi_{1 s}^{(2)}\right),
\end{aligned}
$$

where $A_{g}$ and $A_{u}$ are normalization constants and the superscripts are the nuclear indices. We assume the following coherent superposition of these two states:

$$
\psi(t)=c_{g} e^{-i \omega_{g} t} \psi_{\sigma_{g} 1 s}+c_{u} e^{-i \omega_{u} t} \psi_{\sigma_{u} 1 s}
$$

where $c_{g}$ and $c_{u}$ are the amplitudes of the $\left|\sigma_{g} 1 s\right\rangle$ and $\left|\sigma_{u} 1 s\right\rangle$ states, respectively. (In our model, we do not consider the production of this state or nuclear motion effects.) The elastic scattering amplitude and the elastic DSCS for this superposition state of $T_{2}^{+}$can be obtained in the Born approximation by using procedures similar to those used for the $\mathrm{H}$ atom. In particular, inelastic processes and exchange effects are neglected [19,23]. Since the temporal scales of nuclear rotation and separation for the heavy tritium nuclei are much larger than the temporal width of the electron pulse, treating the nuclear separation as stationary should be a good approximation. The elastic scattering amplitude for fixed $\mathbf{R}$ is

$$
\begin{aligned}
f(\mathbf{q} ; t)= & \frac{4 m}{q^{2}}\left[\left(1+\frac{16\left(\left|c_{g}\right|^{2} A_{g}^{2}+\left|c_{u}\right|^{2} A_{u}^{2}\right)}{\left(4+q^{2}\right)^{2}}\right) \cos \left(\frac{\mathbf{R}}{2} \cdot \mathbf{q}\right)\right. \\
& +\left(\left|c_{g}\right|^{2} A_{g}^{2}-\left|c_{u}\right|^{2} A_{u}^{2}\right) \int d \mathbf{r}^{\prime} \phi_{1 s}^{(1)} \phi_{1 s}^{(2)} e^{-i \mathbf{q} \cdot \mathbf{r}^{\prime}} \\
& \left.+i \frac{16 A_{g} A_{u}\left(c_{g}^{*} c_{u} e^{-i \omega_{u g} t}+\text { c.c. }\right)}{\left(4+q^{2}\right)^{2}} \sin \left(\frac{\mathbf{R}}{2} \cdot \mathbf{q}\right)\right],
\end{aligned}
$$

where $\mathbf{R}$ points from nucleus 1 to nucleus 2 . Uncertainties in both the molecular alignment and the internuclear separation require further consideration. We assume an alignment distribution of the form $\cos ^{12} \theta$ with a half-width of $19.3^{\circ}$; we assume the bond length distribution is Gaussian with a half-width of 5\%. The DSCSs are averaged over these alignment and separation distributions.
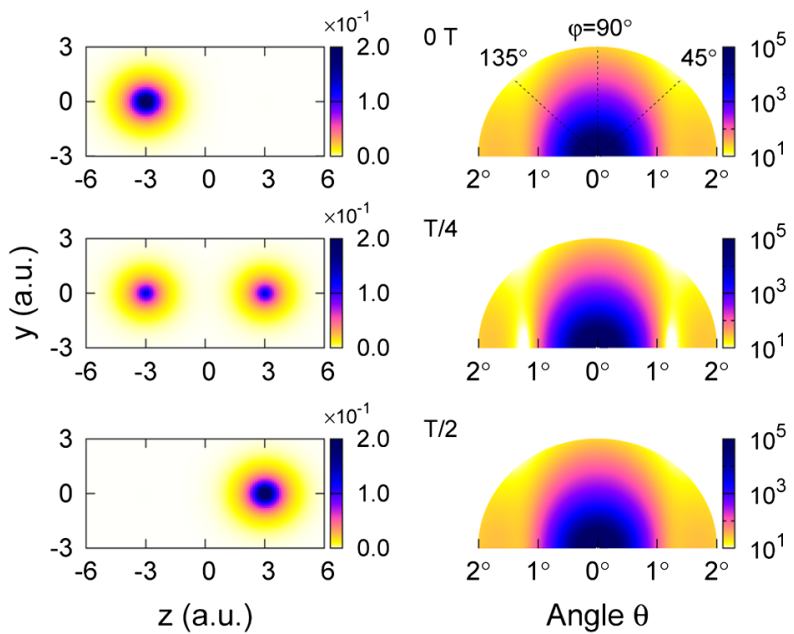

FIG. 5 (color online). Charge density of the $T_{2}^{+}$state in Eq. (9) (with $c_{g}=c_{u}, R=6$ ) in the $y-z$ plane and the corresponding averaged elastic DSCS for three delay times. 

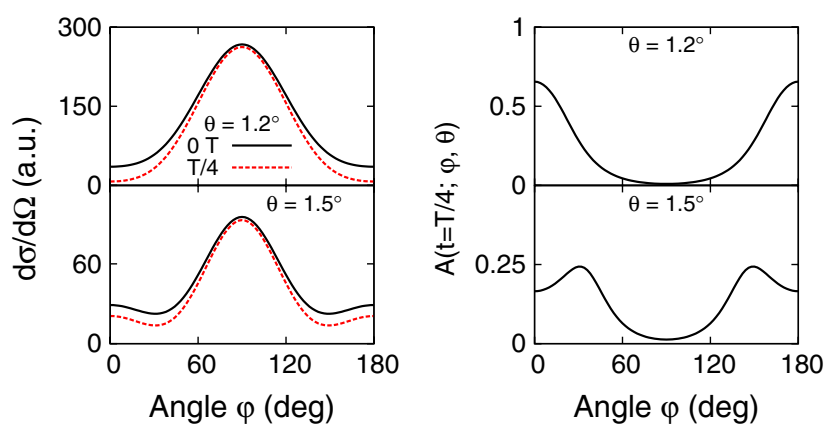

FIG. 6 (color online). Left column: Averaged elastic DSCS (from the right column of Fig. 5) plotted vs azimuthal angle $\varphi$ for two polar angles $\theta$. Right column: The corresponding temporal asymmetry parameter $A(t=\mathrm{T} / 4 ; \varphi, \theta)$ [cf. Eq. (11)].

For the $T_{2}^{+}$superposition state in Eq. (9) having $c_{g}=c_{u}$, Fig. 5 shows the electronic charge density and the corresponding averaged elastic DSCS for three time delays [relative to production of the state (9)], where the parameters of the attosecond electron pulse are the same as for the $\mathrm{H}$ atom case. Owing to negligible overlap of the atomic orbitals for large $\mathbf{R}$, the second term in Eq. (10) is omitted. For $t=0 \mathrm{~T}$ and $\mathrm{T} / 2$ the electron charge density is localized on one nucleus or the other, whereas for $t=\mathrm{T} / 4$ the charge density is delocalized. In the latter case, interference between electron waves scattered from the charge densities at each nucleus results in deep minima in the DSCS, whose locations determine $R$ [7]. For $t=0$ or $\mathrm{T} / 2$, the DSCSs are the same in the asymptotic region; i.e., it is not possible to determine on which nucleus the charge density is localized. In Fig. 6, we plot the DSCS vs azimuthal angle $\varphi$ for two polar angles $\theta$ as well as a temporal asymmetry parameter

$$
A(t ; \varphi, \theta)=\frac{d \sigma(0)-d \sigma(t)}{d \sigma(0)+d \sigma(t)},
$$

for a delay $t$ (where $t=\mathrm{T} / 4$ in Fig. 6). Clearly, the maximum temporal asymmetries occur at particular azimuthal angles. Note also that significant temporal asymmetries occur for large polar angles, where inelastic scattering should be negligible [23].

In conclusion, the DSCSs for ultrafast electron pulse scattering from coherent superposition states of the $\mathrm{H}$ atom and the $T_{2}^{+}$molecule were calculated. They show clearly the "breathing" of the charge density in the $\mathrm{H}$ atom and the localization and delocalization of the charge density in the $T_{2}^{+}$molecule. The present simulations confirm the feasibility of using attosecond electron pulses for 4D investigations of electron dynamics $[15,16,23]$. Such in- vestigations complement those using optical attosecond pulses in providing insights into controlling electronic motion in atoms and molecules.

We gratefully acknowledge informative conversations with M. Centurion concerning experimental capabilities. This work is supported in part by NSF Grant No. PHY0901673 and by a Nebraska Research Initiative grant.

[1] P. B. Corkum and F. Krausz, Nature Phys. 3, 381 (2007).

[2] F. Krausz and M. Ivanov, Rev. Mod. Phys. 81, 163 (2009).

[3] G. Sansone et al., Science 314, 443 (2006).

[4] E. Goulielmakis et al., Science 320, 1614 (2008).

[5] L. Y. Peng, E. A. Pronin, and A. F. Starace, New J. Phys. 10, 025030 (2008).

[6] E. A. Pronin, A.F. Starace, M. V. Frolov, and N.L. Manakov, Phys. Rev. A 80, 063403 (2009).

[7] R. Srinivasan, V. A. Lobastov, C.-Y. Ruan, and A.H. Zewail, Helv. Chim. Acta 86, 1761 (2003).

[8] A. H. Zewail, Annu. Rev. Phys. Chem. 57, 65 (2006).

[9] P. Reckenthaeler, M. Centurion, W. Fuß, S. A. Trushin, F. Krausz, and E.E. Fill, Phys. Rev. Lett. 102, 213001 (2009).

[10] B. Barwick, H. S. Park, O.-H. Kwon, J. S. Baskin, and A. H. Zewail, Science 322, 1227 (2008).

[11] A. Yurtsever and A. H. Zewail, Science 326, 708 (2009).

[12] B. Barwick, D. J. Flannigan, and A.H. Zewail, Nature (London) 462, 902 (2009).

[13] A. H. Zewail, Science 328, 187 (2010).

[14] E. Fill, L. Veisz, A. Apolonski, and F. Krausz, New J. Phys. 8, 272 (2006).

[15] P. Baum and A. H. Zewail, Proc. Natl. Acad. Sci. U.S.A. 104, 18409 (2007).

[16] S. A. Hilbert, C. Uiterwaal, B. Barwick, H. Batelaan, and A. H. Zewail, Proc. Natl. Acad. Sci. U.S.A. 106, 10558 (2009).

[17] P. Hommelhoff, C. Kealhofer, and M. A. Kasevich, Phys. Rev. Lett. 97, 247402 (2006).

[18] H. A. Bethe and R.W. Jackiw, Intermediate Quantum Mechanics (Benjamin, New York, 1968).

[19] R. A. Bonham and M. Fink, High Energy Electron Scattering (Van Nostrand Reinhold, New York, 1974), p. 97.

[20] G. L. Yudin, S. Chelkowski, J. Itatani, A. D. Bandrauk, and P. B. Corkum, Phys. Rev. A 72, 051401(R) (2005).

[21] S. Chelkowski, G. L. Yudin, and A. D. Bandrauk, J. Phys. B 39, S409 (2006).

[22] A. D. Bandrauk, S. Chelkowski, P. B. Corkum, J. Manz, and G. L. Yudin, J. Phys. B 42, 134001 (2009).

[23] P. Baum, J. Manz, and A. Schild, Sci. China Ser. G 53, 987 (2010).

[24] T. E. Sharp, At. Data Nucl. Data Tables 2, 119 (1970). 